\title{
Influência da temperatura de manutenção da fase não-parasitária sobre a fase parasitária de Amblyomma cajennense (Fabricius, 1787) (Acari: Ixodidae)
}

\author{
Influence of the maintenance temperature, of the non parasitic phase, on \\ the parasitic phase, of Amblyomma cajennense \\ (Fabricius, 1787 Acari: Ixodidae)
}

\author{
Samuel Candanedo Chacón, ${ }^{\star}$ F. S. Barbieri, ${ }^{\star}$ P.G. Correia, ${ }^{\star}$ João Luís Horácio Faccini, ${ }^{\star}$ Erik Daemon ${ }^{\star}$
}

\begin{abstract}
Resumo
Com o propósito de avaliar os efeitos de diferentes temperaturas de manutenção da fase não-parasitária sobre a fase parasitária de Amblyomma cajennense, ovos, larvas e ninfas foram mantidos em temperaturas constantes de 18,27 e $32^{\circ} \mathrm{C}$, umidade relativa de $80 \pm 10 \%$ e escotofase. Não houve desenvolvimento embrionário na temperatura de $32^{\circ} \mathrm{C}$, enquanto que, a $18^{\circ} \mathrm{C}$, o percentual de eclosão foi de apenas $3 \%$. Após as mudas das larvas e ninfas mantidas nas três temperaturas, os ínstares subseqüentes foram transferidos para coelhos domésticos e eqüinos, respectivamente. $O$ ciclo de vida do carrapato foi influenciado pela temperatura de manutenção dos ínstares, sendo que a temperatura de $18^{\circ} \mathrm{C}$ prolongou o período parasitário das ninfas e fêmeas quando comparada às temperaturas de 27 e $32^{\circ} \mathrm{C}(\mathrm{p}<0,05)$. Na temperatura de $32^{\circ} \mathrm{C}$, o período ninfal foi aumentado significativamente $(p<0,05)$ em relação à de $27^{\circ} \mathrm{C}$, enquanto as fêmeas apresentaram período parasitário inferior, porém não significativo $(p>0,05)$. O percentual de recuperação das ninfas ingurgitadas não sofreu influência da temperatura de procedência dos exemplares ao nível de $5 \%$ de significância. O peso, tanto de ninfas quanto de fêmeas, foi influenciado pela temperatura de procedência destes ínstares anteriores, sendo que a temperatura de $32^{\circ} \mathrm{C}$ foi a que mais afetou $(p<0,05)$ o peso, já que ínstares procedentes desta temperatura apresentaram as menores médias de peso. A temperatura de $27^{\circ} \mathrm{C}$ foi considerada a mais eficiente para a manutenção da fase não-parasitária. A temperatura de $18^{\circ} \mathrm{C}$ mostrouse adequada para retardar o ciclo biológico de $A$. cajennense, a partir do estágio larval, porém não permite manter uma colônia permanentemente, já que o percentual de desenvolvimento embrionário é muito reduzido. A temperatura de $32^{\circ} \mathrm{C}$ foi considerada a mais deletéria para o ciclo de $A$. cajennense.
\end{abstract}

Palavras-chave: Amblyomma cajennense, temperatura, fase parasitária.

\begin{abstract}
With the purpose of evaluating the effects of different temperatures of maintenance of the free living stages on the parasitic phase of Amblyomma cajennense, eggs, larvae and nymphs were kept in constant temperatures of 18,27 and $32^{\circ} \mathrm{C}$, relative humidity of $80 \pm 10 \%$ and darkness. There was not development of the embryo at $32{ }^{\circ} \mathrm{C}$, and at $18^{\circ} \mathrm{C}$ the hatching rate was $3 \%$. After the ecdisys of the larvae and nymphs maintained in the three temperatures, the subsequent stages were transferred to domestic rabbits and horses, respectively.

The life cycle of the tick was influenced by the temperature of maintenance. The temperature of $18^{\circ} \mathrm{C}$ extendend the parasitic period of the nymphs and females when compared to the temperatures of 27 and $32^{\circ} \mathrm{C}(\mathrm{p}<0.05)$. At $32^{\circ} \mathrm{C}$, the nymphal period was extended $(p<0.05)$ in relation to $27^{\circ} \mathrm{C}$, whereas for females, it was shortened $(p>0.05)$. The rate of recovery of both engorged nymphs and adults was not influenced $(p>0.05)$ by the temperatures of maintenance of the earlier stages.

The weight of engorged nymphs and females was influenced by the temperature of origin of these previous stages. The lowest weights occurred at $32^{\circ} \mathrm{C}(\mathrm{p}<0.05)$. The temperature of $27^{\circ} \mathrm{C}$ was considered the most efficient for the maintenance of the free living phase. The temperature of $18^{\circ} \mathrm{C}$ is appropriate for slowing the life cycle of $A$. cajennense from larvae to adult. However it does not allow to maintain a colony permanently, since the embryogenic development of the eggs is very reduced. The temperature of $32^{\circ} \mathrm{C}$ was considered the most harmful for the life cycle of this tick.
\end{abstract}

Keywords: Amblyomma cajennense, temperature, parasitic phase.

* CPGCV/DPA/IV/UFRRJ, Km 7, BR 465, 23890-000, Seropédica, RJ, Brasil. Email: scchacon@ufrrj.br ou faccini@ufrrj.br 


\section{Introdução}

O carrapato-estrela Amblyomma cajennense (Fabricius, 1787) encontra-se amplamente distribuído no continente americano, desde o sul dos Estados Unidos, México, América Central, Antilhas e em partes da América do Sul, concentrando-se principalmente em áreas ao longo da costa do Oceano Atlântico (Robinson, 1926); leva seu nome específico derivado de Cayenne, lugar da Guiana Francesa onde foi coletado pela primeira vez (United States Department of Agriculture, 1976). No Brasil ocorre durante todos os meses do ano, sendo a forma adulta mais freqüente entre setembro e dezembro (Serra Freire, 1982; Oliveira et al., 2000).

Embora os eqüinos sejam considerados os hospedeiros principais, outras espécies animais podem servir como hospedeiros para os diversos estágios deste ixodídeo (Rohr, 1909; Aragão, 1936; Santos et al., 1985; Linardi et al., 1987). É indispensável salientar a importância do parasitismo de estágios imaturos do carrapato estrela em aves migratórias, as quais atuariam como veículo de dispersão do mesmo e, provavelmente, das doenças por ele transmitidas (Rojas et al., 1999).

A picada deste carrapato causa irritação na pele do hospedeiro, podendo levar a alterações cutâneas (Serra Freire, 1979). É considerado também como indutor de paralisia em ruminantes no Brasil, segundo Serra Freire (1983). Devido ao fato de precisar de três hospedeiros para completar seu ciclo, apresenta maior possibilidade de se infectar e transmitir patógenos (Prata et al., 1997). É considerado transmissor de Babesia equi (Horta e Figueiredo, 1914), Ehrlichiose dos Bovinos (Massard, 1984), Rickettsia rickettsii (Travassos e Vallejo-Freire, 1944; Lemos et al., 1997a, b) agente etiológico da "Febre das Montanhas Rochosas", também conhecida como "Febre Maculosa Paulista" (Santos et al., 1985) e potencialmente capaz de transmitir alguns vírus, como o Flavivírus, ao homem (Figueiredo et al., 1999). Alterações hematológicas em bovinos parasitados foram observadas por Serra Freire e Cunha (1987).

A importância de um conhecimento adequado sobre a influência da temperatura no desenvolvimento dos ixodídeos pode ser exemplificada pela utilização de faixas térmicas na formulação de estratégias de controle (De La Vega e Díaz, 1987; 1992; De La Vega et al., 1988; 1993) ou ainda, no caso de Travassos e Vallejo-Freire (1944), que utilizaram-se da temperatura para melhor administrar as colônias de $A$. cajennense, acelerando ou alongando o ciclo desta espécie de carrapato para facilitar a fabricação da vacina contra Febre Maculosa.

A influência de fatores abióticos sobre as características biológicas intrínsecas de populações desta espécie de carrapato, adaptadas à região neotropical, começaram a ser desvendadas no início da década de 1990 por pesquisadores da UFRRJ, principalmente no que concerne à temperatura (Daemon e Ishizuka 1992, 1995; Prata, 1998) e umidade relativa (Silva et al., 2000). No entanto, algumas lacunas no conhecimento científico ainda existem, como, por exemplo, a relação entre temperatura de manutenção em laboratório das fases de vida livre e período parasitário, objetivo deste experimento. Por outro lado, os estudos que discutem a influência da temperatura de manutenção da fase de vida livre sobre a fase parasitária dos ixodídeos, fator fundamental para o entendimento do ciclo biológico e manutenção de colônias em laboratório, já que as características intrínsecas variam entre populações e entre espécies, restringem-se ao artigo de Bellato e Daemon (1997) com Rhipicephalus sanguineus. Este trabalho faz parte de uma linha de pesquisa mais ampla, ora em desenvolvimento no DPA, e que visa obter dados sobre a biologia das espécies de carrapatos neotropicais.

\section{Material e métodos}

O presente trabalho foi realizado no Laboratório de Ixodologia da Estação para Pesquisas Parasitológicas W. O. Neitz (EPPWON) do Curso de Pós-graduação em Medicina Veterinária - Parasitologia Veterinária, do Departamento de Parasitologia Animal, Instituto de Veterinária, Universidade Federal Rural do Rio de Janeiro (UFRRJ), no período de dezembro de 1999 a dezembro de 2000.

Foram utilizados como hospedeiros para as fases de larva e ninfa exemplares da espécie Oryctolagus cuniculus (L., 1758), coelhos mestiços Califórnia x Nova Zelândia com idade entre 60 e 90 dias, de ambos os sexos, com peso inicial entre 1,5 e $2,1 \mathrm{~kg}$, sem contato prévio com carrapatos e produtos acaricidas, provenientes do Setor de Cunicultura do Instituto de Zootecnia da UFRRJ. Os animais foram mantidos durante o período experimental em gaiolas individuais, em condições ambientais, onde receberam ração comercial para coelhos e água. Para realização da fase parasitária de adultos de $A$. cajennense foram utilizados como hospedeiros eqüinos (Equus cabalus L., 1758), provenientes do Setor de Apreensão da UFRRJ. Os animais foram mantidos durante a fase experimental em baias individuais, em condições ambientais, onde receberam capim picado e água.

Fêmeas ingurgitadas de $A$. cajennense com peso médio de $794,07 \pm 175,9 \mathrm{mg}$ foram coletadas de eqüinos naturalmente infestados e sem contato recente com carrapaticidas, no município de Seropédica (Lat.: $22^{\circ} 45^{\prime}$ 'S; Long.: $43^{\circ} 41^{\prime} \mathrm{W}$; Alt.: $33 \mathrm{~m})$, estado do Rio de Janeiro, Brasil. Estas foram transportadas ao laboratório, limpas, pesadas, identificadas, acondicionadas em placas de Petri por fixação em posição dorsal com auxílio da fita adesiva e mantidas sob condições controladas em câmara climatizada [temperatura de $27 \pm 1^{\circ} \mathrm{C}$, umidade relativa (UR) de $80 \pm 10 \%$ e escotofase], para a realização da postura (Prata, 1998).

A postura foi acompanhada e os ovos de cada fêmea coletados de três em três dias após o início da postura, reunidos, misturados e em grupos de $100 \mathrm{mg}$ acondicionados em seringas plásticas descartáveis com capacidade de $5 \mathrm{ml}$, previamente preparadas e identificadas. Estes ovos foram incubados nas mesmas condições controladas descritas para as fêmeas ingurgitadas. Foi feita a verificação contínua dos ovos, até a eclosão total das larvas.

Um grupo de 18 coelhos foi infestado através da técnica do saco de pano aderido à base das orelhas (Neitz et al., 1971; Cunha, 1978). Cada coelho recebeu uma dose infestante de larvas, aproximadamente 3280 larvas equivalentes a eclosão total de 200mg de ovos (Prata e Daemon, 1997), com 15 a 25 dias de jejum. Foi feita a coleta diária das larvas ingurgitadas que se desprenderam após a fase parasitária. No laboratório estas larvas foram limpas, pesadas, misturadas e acondicionadas em seringas plásticas de $10 \mathrm{ml}$ contendo $764 \mathrm{mg}$ de larvas por seringa, equivalente a 1000 larvas ingurgitadas (Prata et al., 1998). 
A partir das larvas coletadas, todo o desenvolvimento da fase não-parasitária foi realizado separadamente em câmaras climatizadas $(B O D)$, reguladas em três temperaturas diferentes $-18,27$ e $32, \pm 1^{\circ} \mathrm{C}$ - como descrito por Bellato e Daemon (1997). As temperaturas extremas correspondem às médias das máximas e das mínimas da região onde foi realizado o estudo, enquanto a temperatura de $27^{\circ} \mathrm{C}$ é a comumente utilizada para o desenvolvimento dos carrapatos das regiões neotropicais (Daemon e Ishizuka, (1992). Para as três temperaturas, a UR foi de $80 \pm 10 \%$ e escotofase. Para cada temperatura foram colocadas na câmara climatizada 15 seringas e feita a observação contínua dos ínstares para verificação do fim da fase não-parasitária e para confirmação da emergência ninfal.

Após sete a dez dias da ecdise ninfal, ninfas provenientes da muda de $764 \mathrm{mg}$ de larvas ingurgitadas [aproximadamente 1000 ninfas (Prata et al., 1998)] foram utilizadas para as infestações por coelho, repetindo a metodologia descrita para as larvas. Utilizando sete coelhos para a temperatura de $18^{\circ} \mathrm{C}$, quatro para as ninfas provenientes de $27^{\circ} \mathrm{C}$ e cinco para as provenientes de $32^{\circ} \mathrm{C}$. Após a fase parasitária, a coleta das ninfas ingurgitadas foi realizada como descrito anteriormente, contando e pesando as ninfas por tratamento, coelho e dia, calculando as médias de peso por tratamento. As ninfas então foram acondicionadas em seringas de $10 \mathrm{ml}$ contendo aproximadamente 80 exemplares e colocadas nas mesmas temperaturas de procedência em câmara climatizada para realização da fase não-parasitária. Após observada a emergência dos adultos e verificado o percentual de ecdise, foram realizadas as infestações, utilizando-se eqüinos como hospedeiros.

Para realização da fase parasitária de adultos foram utilizados 160 casais de A. cajennense com 15 dias de jejum, por eqüino, sendo dois animais para cada temperatura, independentemente do sexo, uma vez que nada há na literatura com relação à influência do sexo do hospedeiro no período parasitário dos adultos desta espécie. Para cada animal foram utilizados 80 casais de $A$. cajennense de cada lado do pescoço, utilizando uma adaptação da técnica descrita por Sanavria e Prata (1996).

Depois de observada a fase parasitária das fêmeas oriundas de cada tratamento, estas foram recuperadas, limpas, pesadas, identificadas e colocadas para postura nas suas temperaturas de origem. Assim, foi acompanhado o processo de postura das fêmeas ingurgitadas oriundas dos cavalos nas três temperaturas. Os ovos foram pesados a cada três dias, sendo depois misturados e separados em amostras contendo $100 \mathrm{mg}$, acondicionados em seringas plásticas e incubados nas mesmas temperaturas de origem.

Após a incubação dos ovos, foi observada a eclosão das larvas. Assim, após 20 a 30 dias de jejum, foi feita a infestação em cinco coelhos, com o equivalente da eclosão total de $100 \mathrm{mg}$ de ovos, aproximadamente 1.640 larvas, e observada a fase parasitária das larvas.

As atividades relacionadas com o acompanhamento da fase experimental foram realizadas diariamente pela manhã, entre 8 e 10 horas.

A análise da fase parasitária de larvas, ninfas e fêmeas sob influência de diversas temperaturas foi realizada levando em consideração:

- Período parasitário - compreendido desde a infestação até o desprendimento e coleta dos ínstares ingurgitados (Bellato e Daemon, 1997).

- Ritmo de recuperação - seqüência diária de desprendimento dos exemplares.

- Percentual de recuperação - total dos exemplares ingurgitados coletados em relação ao total de indivíduos utilizados nas infestações (Bellato e Daemon, 1997).

Não foi possível calcular o percentual de recuperação das fêmeas alimentadas no eqüino com exatidão, já que a técnica de infestação é muito difícil de ser aplicada sem perdas, como descrito por Sanavria et al., (1996).

- Peso dos exemplares ingurgitados.

Para comparação estatística foram utilizados a Análise de Variância (ANOVA) e o teste Tukey-Kramer, do programa estatístico Instat.

\section{Resultados e discussão}

\section{Fase parasitária de ninfas}

Os parâmetros biológicos e os resultados da análise estatística da fase parasitária de ninfas de $A$. cajennense, alimentadas em coelhos, estão impressos na Tabela 1.

Tabela 1 - Período parasitário, porcentagem de recuperação e peso de ninfas ingurgitadas de Amblyomma cajennense, coletados de 16 coelhos infestados artificialmente com 1000 ninfas cada, procedentes de 18,27 e $32^{\circ} \mathrm{C}$, UR de $80 \pm 10 \%$ e escotofase

\begin{tabular}{|c|c|c|c|c|}
\hline Parâmetros & $\begin{array}{c}\text { Medidas de } \\
\text { Tendência Central }\end{array}$ & $18 \pm 1^{\circ} \mathrm{C}$ & $27 \pm 1^{\circ} \mathrm{C}$ & $32 \pm 1^{\circ} \mathrm{C}$ \\
\hline $\begin{array}{c}\text { Periodo Parasitário } \\
\text { (dias) }\end{array}$ & $\begin{array}{c}\text { Extremos } \\
\bar{x} \pm S D \\
n(\text { ninfas })\end{array}$ & $\begin{array}{c}3-7 \\
4,84^{a} \pm 0,80 \\
5702\end{array}$ & $\begin{array}{c}3-7 \\
4,25^{b} \pm 0,87 \\
3371\end{array}$ & $\begin{array}{c}3-8 \\
4,53^{\mathrm{c}} \pm 0,91 \\
3924\end{array}$ \\
\hline $\begin{array}{l}\text { Percentual de } \\
\text { Recuperação }\end{array}$ & $\begin{array}{c}\text { Extremos } \\
\bar{x} \pm \mathrm{SD} \\
\mathrm{n} \text { (coelhos) }\end{array}$ & $\begin{array}{c}53,49-98,01 \\
81,42^{\mathrm{a}} \pm 15,48 \\
7 \\
\end{array}$ & $\begin{array}{c}64,14-92,99 \\
84,28^{a} \pm 13,50 \\
4 \\
\end{array}$ & $\begin{array}{c}73,31-91,05 \\
78,48^{\mathrm{a}} \pm 7,61 \\
5 \\
\end{array}$ \\
\hline Peso (mg) & $\begin{array}{c}\text { Extremos } \\
\bar{x} \pm S D \\
n \text { (amostras) }\end{array}$ & $\begin{array}{c}7,75-16,57 \\
13,89^{a} \pm 2,03 \\
29\end{array}$ & $\begin{array}{c}10,76-15,88 \\
13,42^{\mathrm{a}} \pm 1,66 \\
16\end{array}$ & $\begin{array}{c}8,3-16,37 \\
11,96^{\mathrm{b}} \pm 2,07 \\
25\end{array}$ \\
\hline
\end{tabular}

Médias seguidas de letras iguais não diferem entre si ao nível de 0,05 . ' $x$ =Média; SD=Desvio-padrão.

Foi observado um aumento no período parasitário nos exemplares procedentes das temperaturas de 18 e $32^{\circ} \mathrm{C}$ em relação à temperatura de $27^{\circ} \mathrm{C}$, sendo a temperatura de $18^{\circ} \mathrm{C}$ a que mais prolongou a fase parasitária das ninfas $(p<0,05)$. $O$ ritmo de recuperação dos exemplares apresentou variação 
conforme a temperatura de procedência. Nas temperaturas de 27 e $32^{\circ} \mathrm{C}$, o pico máximo de recuperação ocorreu no quarto dia de parasitismo com declínio no quinto dia, enquanto os exemplares oriundos da temperatura de $18^{\circ} \mathrm{C}$ apresentaram o pico máximo de recuperação no quinto dia, prolongando o periodo parasitário. $O$ percentual médio de recuperação não teve variação significativa ( $p>0,05)$, onde o maior e o menor percentuais médios foram observados em ninfas provenientes de 27 e $32^{\circ} \mathrm{C}$, respectivamente. O percentual de recuperação acumulado foi semelhante nas três temperaturas. Embora a diferença entre os percentuais de recuperação não tenha sido estatisticamente significante, a diferença de aproximadamente três pontos percentuais entre as temperaturas de 18 e $27^{\circ} \mathrm{C}$ e seis pontos percentuais entre as temperaturas de 32 e $27^{\circ} \mathrm{C}$, sugerem ser a temperatura de $27^{\circ} \mathrm{C}$ a mais indicada para manutenção da fase não-parasitária das ninfas. As médias dos pesos das ninfas recuperadas também variaram conforme a temperatura de procedência, sendo que as ninfas mais pesadas foram provenientes de 18 e $27^{\circ} \mathrm{C}$. A análise estatística demonstrou diferença significativa $(p<0,05)$ entre o peso das ninfas recuperadas na temperatura de $32^{\circ} \mathrm{C}$ e daquelas recuperadas nas temperaturas de 18 e $27^{\circ} \mathrm{C}$. Não houve diferença significativa para os pesos nestas temperaturas $(p>0,05)$.

Pode se afirmar que as diferenças encontradas em relação aos parâmetros analisados (Tabela 1) no presente trabalho estão relacionadas com a temperatura de procedência dos exemplares. Este efeito não relatado na literatura consultada para esta espécie, provavelmente decorre da ação de diferentes temperaturas sobre a fisiologia do carrapato e está relacionado com características intrínsecas desta espécie.

Bellato e Daemon (1997) encontraram diferença significativa para os períodos parasitários de ninfas de Rhipicephalus sanguineus provenientes das temperaturas de 18,27 e $32^{\circ} \mathrm{C}$, observando um aumento no período parasitário dos ínstares procedentes da temperatura menor para a mais elevada e um maior e menor peso das ninfas nas temperaturas de 27 e $18^{\circ} \mathrm{C}$, respectivamente, dados que apresentaram tendências diferentes dos dados encontrados para $A$. cajennense no presente experimento. Estas diferenças se devem, provavelmente, a diferenças interespecíficas, podendo deste modo representar adaptações às diversas condições climáticas que as espécies experimentam nas suas extensões geográficas, como relatado por Chilton et al., (2000) para as espécies Amblyomma limbatum e Aponomma hidrosauri, parasitos de lagartos no continente australiano.

\section{Fase parasitária de fêmeas}

Os parâmetros biológicos e os resultados da análise estatística da fase parasitária de fêmeas de $A$. cajennense estão apresentados na Tabela 2.

Foi observado um aumento significativo $(p<0,05)$ no período parasitário nos exemplares procedentes da temperatura de $18^{\circ} \mathrm{C}$ em relação aos procedentes das temperaturas de 27 e $32^{\circ} \mathrm{C}$. O

' $x$ =Média;

$\mathrm{SD}=$ Desvio-padrão. ritmo de recuperação das fêmeas apresentou variação conforme a temperatura de procedência. Na temperatura de $27^{\circ} \mathrm{C}$, o início de recuperação ocorreu no sétimo dia de infestação, com um pico no nono dia e se estendeu até o décimo sétimo dia. $\mathrm{Na}$ temperatura de $32^{\circ} \mathrm{C}$ houve um encurtamento do período de recuperação, com início no oitavo dia, pico no décimo dia e final de recuperação no décimo quarto dia de parasitismo, enquanto os exemplares provenientes da temperatura de $18^{\circ} \mathrm{C}$ apresentaram início de recuperação no décimo dia, picos de recuperação no décimo segundo e décimo terceiro dias, prolongando o parasitismo até o vigésimo dia. O maior percentual de recuperação foi observado em fêmeas provenientes de $27^{\circ} \mathrm{C}$. O percentual de recuperação acumulado das fêmeas foi semelhante nas três temperaturas, apresentando variações em decorrência das variações no ritmo de recuperação. À semelhança do ocorrido com as ninfas, o maior percentual de recuperação ocorreu a $27^{\circ} \mathrm{C}$, indicando ser esta a temperatura que melhor se adequa à manutenção das fêmeas não ingurgitadas. O peso médio das fêmeas foi maior nos ínstares provenientes do tratamento a $27^{\circ} \mathrm{C}$, enquanto os menores pesos foram observados no tratamento de $32^{\circ} \mathrm{C}$, o qual foi estatisticamente diferente $(p<0,05)$ dos outros dois.

Bellato e Daemon (1997) encontraram uma relação temperatura-período parasitário para $R$. sanguineus semelhante à encontrada no presente trabalho, ocorrendo um aumento do período parasitário das fêmeas que se originaram de ninfas provenientes de $18^{\circ} \mathrm{C}$. Ainda segundo os autores mencionados, o percentual de recuperação não apresentou diferença significativa nesta espécie. No entanto, fêmeas de $R$. sanguineus criadas a $27^{\circ} \mathrm{C}$ e $32^{\circ} \mathrm{C}$ obtiveram médias de peso maior do que as fêmeas provenientes de $18^{\circ} \mathrm{C}$, cujo peso foi estatisticamente diferente das provenientes de 27 e $32^{\circ} \mathrm{C}$. Estes dados são parcialmente diferentes dos encontrados por nós, já que fêmeas de $A$. cajennense apresentaram maior peso quando mantidas a $27^{\circ} \mathrm{C}$, porém menor quando mantidas a $32^{\circ} \mathrm{C}$. A diferença na sensibilidade das fêmeas das duas espécies a diferentes temperaturas está, provavelmente, também relacionada com a variação interespecífica e é conseqüência dos processos adaptativos das várias espécies, como já discutido na seção referente a ninfas.

Tabela 2 - Período parasitário, porcentagem de recuperação e peso de fêmeas ingurgitadas de Amblyomma cajennense, coletadas de seis eqüinos (dois/tratamento), infestados artificialmente com 160 casais cada, procedentes de 18,27 e $32^{\circ} \mathrm{C}$, UR de $80 \pm 10 \%$ e escotofase

\begin{tabular}{|c|c|c|c|c|}
\hline Parâmetros & $\begin{array}{c}\text { Medidas de } \\
\text { Tendência Central }\end{array}$ & $18 \pm 1^{\circ} \mathrm{C}$ & $27 \pm 1^{\circ} \mathrm{C}$ & $32 \pm 1^{\circ} \mathrm{C}$ \\
\hline $\begin{array}{l}\text { Periodo Parasitário } \\
\text { (dias) }\end{array}$ & $\begin{array}{c}\text { Extremos } \\
\bar{x} \pm S D \\
n \text { (fêmeas) } \\
\end{array}$ & $\begin{array}{c}10-20 \\
13,5^{a} \pm 1,85 \\
118\end{array}$ & $\begin{array}{c}7-17 \\
10,55^{b} \pm 2,23 \\
185 \\
\end{array}$ & $\begin{array}{c}8-14 \\
10,45^{b} \pm 1,54 \\
99 \\
\end{array}$ \\
\hline $\begin{array}{l}\text { Percentual de } \\
\text { Recuperação }\end{array}$ & $\begin{array}{c}\bar{x} \\
\mathrm{n}(\text { cavalos) } \\
\end{array}$ & $\begin{array}{c}36,87 \\
2 \\
\end{array}$ & $\begin{array}{c}50,94 \\
2 \\
\end{array}$ & $\begin{array}{c}30,94 \\
2 \\
\end{array}$ \\
\hline Peso (mg) & $\begin{array}{c}\text { Extremos } \\
\bar{x} \pm S D \\
n \text { (fêmeas) }\end{array}$ & $\begin{array}{c}55-976,1 \\
557,03^{\mathrm{a}} \pm 198,03 \\
118 \\
\end{array}$ & $\begin{array}{c}94-999,7 \\
575,1^{\mathrm{a}} \pm 166,46 \\
185\end{array}$ & $\begin{array}{c}202,6-788,1 \\
477,21^{b} \pm 101,59 \\
99\end{array}$ \\
\hline
\end{tabular}

Médias seguidas de letras iguais não diferem entre si ao nível de 0,05. 


\section{Fase parasitária de larvas}

Não houve continuidade do ciclo à temperatura de $32^{\circ} \mathrm{C}$, já que não houve eclosão; os ovos ressecaram e encarquilharam. Quando os ovos foram mantidos na temperatura de $27^{\circ} \mathrm{C}$, o ciclo teve continuidade, eclodiram ovos de todas as $36(100 \%)$ seringas colocadas para incubação e o período parasitário das larvas teve duração de três a sete dias com média de 4,28 $\pm 1,07$ dias $(n=2.071)$. Observou-se um pico de recuperação no quarto dia de parasitismo, percentual de recuperação de $25,25 \pm 13,8 \%(15-48,66)(n=$ $5)$ e peso de $0,63 \pm 0,1 \mathrm{mg}(0,42-0,76)(n=23)$. Os ovos mantidos na temperatura de $18^{\circ} \mathrm{C}$ eclodiram em 49 das 51 $(98 \%)$ seringas utilizadas, porém com percentuais muito baixos ( $3 \pm 2,94 \%$ de eclosão), sendo que $77,24 \pm 13,27 \%$ da massa de ovos sofreu encarquilhamento e as larvas eclodidas apresentaram comportamento anormal, ficando no fundo da seringa imóveis ou com pouco movimento, aparentando estar debilitadas e sem mostrar resposta a estímulos externos.

\section{Referências}

ARAGÃO, H. B. Ixodidas brasileiros e de alguns paizes limitrophes. Memórias do Instituto Oswaldo Cruz, v. 31, n. 4, p. 759-845, 1936.

BELLATO, V., DAEMON, E. Influência da temperatura de manutenção da fase não-parasitária sobre a fase parasitária de Rhipicephalus sanguineus (Latreille, 1806) (Acari: Ixodidae). Revista Brasileira de Parasitologia Veterinária., v. 6, n.1, p. 15-19, 1997.

CHILTON, N. B., ANDREWS, R. H., BULL, C. M. Influence of temperature and relative humidity on the moulting success of Amblyomma limbatum and Aponomma hydrosauri (Acari: Ixodidae) larvae and nymphs. International Journa I for Parasitology, v. 30, p. 973-979, 2000.

CUNHA, D.W. Estudos da toxicidade de alguns carrapatos comumente encontrados no Brasil. 1978.78 f. Tese (Mestrado) - Universidade Federal Rural do Rio de Janeiro.

DAEMON, E., ISHIZUKA, A. C. Efeito de diferentes temperaturas sobre a ecdise larval de Amblyomma cajennense (Fabricius, 1787) (Acarina : Ixodidae). Rev. Brasileira de Parasitologia Veterinária., v. 1, n. 2, p. 105-107, 1992.

DAEMON, E., ISHIZUKA, A. C. Efeitos de diferentes temperaturas sobre a ecdise ninfal de Amblyomma cajennense (Acarina : Ixodidae). Rev. Brasileira Ciência Veterinária, v. 2, n. 1, p. 7-9, 1995.

De La VEGA, R., DÍAZ, G. Aplicación de las constantes térmicas en el control de la garrapata del ganado vacuno (Boophilus microplus). V. Supervivencia larvaria en el laboratorio. Rev. Salud Animal, v. 9, p. 259-265, 1987.

De La VEGA, R. , DÍAZ, G. Aplicación de las constantes térmicas en el control de la garrapata del ganado vacuno (Boophilus microplus). VIII. Validación del programa de cuarentena. Rev. Salud Animal, v. 14, p. 133-136, 1992.

De La VEGA, R., FARRADÁ, F., DÍAZ, G. Aplicación de las constantes térmicas en el control de la garrapata del ganado vacuno (Boophilus microplus). VI. Cuarentena. Rev. Salud Animal, v. 10, p. 71-75, 1988.

De La VEGA, R., FARRADÁ, F. , DÍAZ, G. Aplicación de las constantes térmicas en el control de la garrapata del ganado vacuno (Boophilus microplus). VII. Despoblamiento. Rev. Salud Animal, v. 15, p. 162-171, 1993.

FIGUEIREDO, L. T. M., BADRA, S. J., PEREIRA, L. E., SZABÓ, M. P. J. Report on ticks collected in the Southeast and Mid-West regions of Brazil: analysing the potential transmission of tick - borne pathogens to man. Rev. da Sociedade Brasileira de Medicina Tropical, v. 32, n. 6, p. 613-619, 1999.
Embora a temperatura de $18^{\circ} \mathrm{C}$ tenha se mostrado adequada para retardar o ciclo biológico de $A$. cajennense, a partir do estágio larval, conforme observaram Travassos e Vallejo-Freire (1944), ela não permite a manutenção de uma colônia, já que o percentual de desenvolvimento dos embriões é muito reduzido. Neste caso, há necessidade de se combinar as temperaturas de 18 e $27^{\circ} \mathrm{C}$, esta última para permitir o desenvolvimento dos ovos.

Provavelmente, a temperatura de manutenção da fase nãoparasitária de $A$. cajennense afetou o metabolismo que regula as atividades biológicas do carrapato, como descrito por Chilton et al., 2000 para a fase não-parasitária dos carrapatos por eles estudados. O encarquilhamento dos ovos a 18 e $32^{\circ} \mathrm{C}$, pode ter decorrido da ação da temperatura sobre as características físico-químicas da casca do ovo, que não conseguiu manter suas propriedades, alteradas também em função do metabolismo do carrapato, como descrito anteriormente.

HORTA, P. P., FIGUEIREDO, A. S. Nutaliose dos eqüideos em Minas Gerais ("A mijadeira" dos poldrinhos). Rev. Veterinária de Zoociências. v. 4, n. 1, p. 3, 1914.

LEMOS, E. R.D., MACHADO, R.D., COURA, J. R., GUIMARÃES, M. A. A., SERRA FREIRE, N. M., AMORIM, M., GAZETA, G. S. Epidemiological aspects of the Brazilian Spotted Fever: seasonal activity of ticks collected in an endemic area in São Paulo, Brazil. Rev. Sociedade Brasileira Medicina Tropical, v. 30, n. 3, p. 181-185, 1997a.

LEMOS, E. R. D., MACHADO, R. D., PIRES, F. D., MACHADO, S. L., COSTA, L. M., COURA, J. R. Rickettsiae - infected ticks in an endemic area of Spotted Fever in the State of Minas Gerais, Brazil. Mem. Inst. Oswaldo Cruz, v. 92, n. 4, p. 477-481, 1997b.

LINARDI, P. M., TEIXEIRA, V. P., BOTELHO, J. R., RIBEIRO, L. S. Ectoparasitos de roedores em ambientes silvestres do município de Juiz de Fora, Minas Gerais. Mem. Inst. Oswaldo Cruz, v. 82, n. 1, p. 137-139, 1987.

MASSARD, C. A. Ehrlichia bovis (Donatien e Lestoquard, 1936) diagnóstico, cultivo in vitro e aspectos epidemiológicos em bovinos no Brasil. 1984.113 f. Tese (Doutorado) - Universidade Federal Rural do Rio de Janeiro.

NEITZ, W.O., BOUGHTON, F., WALTERS, H.S. Laboratory investigations on the life cycle of the karoo paralysis tick (Ixodes rubicundus Neumann, 1904). Onderstepoort Journal of Veterinary Research, v. 38, n. 3, p. 215-224, 1971.

OLIVEIRA, P. R., BORGES, L. M. F., LOPES, C. M. L., LEITE, R. C. Population dynamics of the free-living stages of Amblyomma cajennense (Fabricius, 1787) (Acari: Ixodidae) on pastures of Pedro Leopoldo, Minas Gerais State, Brazil. Veterinary Parasitology, v. 92, p. 295-301, 2000.

PRATA, M. C. A., ALONSO, L. S. , SANAVRIA, A. Parâmetros biológicos do estágio larval de Amblyomma cajennense (Fabricius, 1787) (Acari: Ixodidae) em coelhos. Rev. Brasileira de Ciência Veterinária, v. 4, n. 1, p. 5-8, 1997.

PRATA, M. C. A. , DAEMON, E. Determinação do número de ovos por grama de postura de Amblyomma cajennense(Fabricius, 1787) (Acari: Ixodidae). Rev. Brasileira de Ciência Veterinária, v. 4, n. 2, p. 81-82, 1997.

PRATA, M. C. A. Efeitos de diferentes temperaturas sobre os processos de postura, eclosão e mortalidade de larvas de Amblyomma cajennense (Fabricius, 1787) (Acari: Ixodidae). 1998. 75 f. Dissertação (Mestrado) - Universidade Federal Rural do Rio de Janeiro. 
PRATA, M. C. A., FACCINI, J. L. H., DAEMON, E. Relationship between weight and number of engorged Amblyomma cajennense larvae and nymphs (Fabricius, 1787) (Acari: Ixodidae) in experimental infestations on rabbits. Rev. Brasileira de Parasitologia Veterinária, v. 7, n. 2, p. 107-111, 1998.

ROBINSON, L. E. Ticks. A Monograph of The Ixodoidea. Part IV. The Genus Amblyomma. Cambridge Univ. Press, 1926. 302 p., pls. I-VII. ROHR, C.J. Estudos sobre Ixodidas do Brasil. 1909. 220 f. Tese (Doutorado) - Instituto Oswaldo Cruz.

ROJAS, R., MARINI, A. M., COUTINHO, Z. T. M. Wild birds as hosts of Amblyomma cajennense (Fabricius, 1787) (Acari: Ixodidae). Mem. Inst. Oswaldo Cruz, v. 94, n. 3, p. 315-322, 1999.

SANAVRIA, A. , PRATA, M. C. A. Metodologia para colonização do Amblyomma cajennense (Fabricius, 1787) (Acari: Ixodidae) em laboratório. Rev. Brasileira de Parasitologia Veterinária, v. 5, n. 2, p. 87-90, 1996. SANAVRIA, A., PRATA, M. C. A., MORAIS, M. C. , ALONSO, L. S. Determinação de alguns parâmetros biológicos de Amblyomma cajennense (Fabricius, 1787) (Acari: Ixodidae) em infestação artificial de eqüinos. Arquivos da Faculdade de Veterinária da Universidade Federal do Rio Grande do Sul, v. 24, n. 2, p. 79-86, 1996.

SANTOS, O. L., MACHADO, R. Z., ALESSI, A. C., BECHARA, G. H., COSTA, A. J. , ROCHA, U. F. Ecologia de carrapatos. XII. Mamíferos domésticos parasitados por Amblyomma cajennense (Fabricius, 1787) em Jaboticabal e Matão, SP. Biológico, v. 51, n. 8, p. 215-218, 1985.
SERRA FREIRE, N. M. Toxicidade de Amblyomma cajennense para ruminantes domésticos e sua significação como agente de uma nova forma de "Tick paralysis". 1979. 119 f. Tese (Doutorado) - Universidade Federal Rural do Rio de Janeiro.

SERRA FREIRE, N. M. Epidemiologia de Amblyomma cajennense: Ocorrência estacional e comportamento dos estádios não-parasitários em pastagens do Estado do Rio de Janeiro. Arquivos da Universidade Federal Rural do Rio de Janeiro, v. 5, n. 2, p. 187-193, 1982.

SERRA FREIRE, N. M. Tick Paralysis in Brazil. Tropical Animal Health Proceedings., v. 15, p. 124-126, 1983.

SERRA FREIRE, N. M. , CUNHA, D. W. Amblyomma cajennense: Comportamento de ninfas e adultos como parasitos de bovinos. Rev. Brasileira de Medicina Veterinária, v. 9, n. 5, p. 100-103, 1987.

SILVA, C.L.G., DAEMON, E., SANTOS, A.C.G. , FACCINI, J.L.H. Efeito de diferentes teores de umidade relativa sobre fêmeas ingurgitadas, ovos e larvas em jejum de Amblyomma cajennense (Acari: Ixodidae). Rev. Universidade Rural Série Ciências da Vida, v. 22 (supl.), p. 137142, 2000.

TRAVASSOS, J., VALLEJO-FREIRE, A. Criação artificial de Amblyomma cajennense para o preparo da vacina contra a febre maculosa. Memórias do Instituto Butantan, v. 18, p. 145-235, 1944.

UNITED STATES DEPARTMENT OF AGRICULTURE. Ticks of Veterinary Importance. Animal and Plant Health Inspection Service, Agriculture Handbook, n. 485, 122 f., 1976. 